\title{
A comparison of thick-film microscopy, rapid … diagnostic test, and polymerase chain reaction for accurate diagnosis of Plasmodium falciparum malaria
}

Kenji O. Mfuh 1,3, Olivia A. Achonduh-Atijegbe ${ }^{3}$, Obase N. Bekindaka ${ }^{3}$, Livo F. Esemu ${ }^{3}$, Calixt D. Mbakop ${ }^{4}$ Krupa Gandhi ${ }^{5}$, Rose G. F. Leke ${ }^{3}$, Diane W. Taylor ${ }^{1}$ and Vivek R. Nerurkar ${ }^{1,2^{*}}$

\begin{abstract}
Background: Accurate diagnosis of malaria is important for effective disease management and control. In Cameroon, presumptive clinical diagnosis, thick-film microscopy (TFM), and rapid diagnostic tests (RDT) are commonly used to diagnose cases of Plasmodium falciparum malaria. However, these methods lack sensitivity to detect low parasitaemia. Polymerase chain reaction (PCR), on the other hand, enhances the detection of sub-microscopic parasitaemia making it a much-needed tool for epidemiological surveys, mass screening, and the assessment of interventions for malaria elimination. Therefore, this study sought to determine the frequency of cases missed by traditional methods that are detected by PCR.
\end{abstract}

Methods: Blood samples, collected from 551 febrile Cameroonian patients between February 2014 and February 2015, were tested for P. falciparum by microscopy, RDT and PCR. The hospital records of participants were reviewed to obtain data on the clinical diagnosis made by the health care worker.

Results: The prevalence of malaria by microscopy, RDT and PCR was 31\%, 45\%, and 54\%, respectively. However, of the $92 \%$ of participants diagnosed as having clinical cases of malaria by the health care worker, $38 \%$ were malarianegative by PCR. PCR detected $23 \%$ and $12 \%$ more malaria infections than microscopy and RDT, respectively. A total of 128 (23\%) individuals had sub-microscopic infections in the study population. The sensitivity of microscopy, RDT, and clinical diagnosis was 57\%, 78\% and 100\%; the specificity was 99\%, 94\%, and 17\%; the positive predictive values were $99 \%, 94 \%$, and $59 \%$; the negative predictive values were $66 \%, 78 \%$, and $100 \%$, respectively. Thus, $41 \%$ of the participants clinically diagnosed as having malaria had fever caused by other pathogens.

Conclusions: Malaria diagnostic methods, such as TFM and RDT missed 12-23\% of malaria cases detected by PCR. Therefore, traditional diagnostic approaches (TFM, RDT and clinical diagnosis) are not adequate when accurate epidemiological data are needed for monitoring malaria control and elimination interventions.

Keywords: Malaria, Diagnosis, PCR, Microscopy, Clinical diagnosis

\footnotetext{
*Correspondence: nerurkar@hawaii.edu

${ }^{1}$ Department of Tropical Medicine, Medical Microbiology and Pharmacology, John A. Burns School of Medicine, University of Hawaii at Manoa, Honolulu, HI, USA

Full list of author information is available at the end of the article
} 


\section{Background}

Malaria remains a major public health threat, particularly in sub-Saharan Africa, where about 191 million new infections and 395,000 deaths were reported in 2015 [1]. The World Health Organization (WHO) now recommends a confirmatory diagnosis of malaria using microscopy and/or RDT before initiation of treatment, partly influenced by the fear of the development of drug resistance and to enable the identification of malaria-negative patients, for which further investigations need to be sought for appropriate treatment [2]. Accurate diagnosis of malaria is thus vital for effective management and control of malaria while avoiding the wrong use of anti-malarial drugs. In most malaria-endemic countries, malaria is usually diagnosed by microscopy or rapid diagnostic tests (RDT) and clinical evidence.

The traditional practice by health care workers $(\mathrm{HCW})$ in malaria-endemic countries has been to diagnose malaria based on a history of fever (clinical diagnosis) [3-6]. The specificity of clinical diagnosis of malaria is reduced by the overlap of malaria symptoms with other tropical diseases, such as typhoid fever, respiratory tract infections, bacterial disease and viral infections. The accuracy of clinical diagnosis may vary with the level of malaria endemicity, malaria transmission season and age group. Malaria microscopy is complex, which includes different species and blood stages of the Plasmodium parasite and requires a competent microscopist. Also, the presence of sub-microscopic parasitaemia greatly reduces the accuracy of malaria diagnosis by TFM. Unlike TFM,
RDT detect malaria antigens, not malaria parasites, which gives them an added advantage in the ability to diagnose malaria in patients with low-grade parasitaemia below the detection limit of TFM $[7,8]$. However, the specificity of the commonly used RDT that detects histidine rich protein-II (HRP-II) of P. falciparum, is limited when the parasite is cleared and antigens remain in circulation for about 28 days (false positive) [2].

As malaria-endemic countries move towards malaria elimination, there is a need for rapid and accurate diagnostic tools for malaria. Active monitoring of the performance of various diagnostic methods for malaria at the country level is necessary to guide policy on the diagnostic methods to use for malaria diagnosis and elimination. In this study, the performance of RDT, TFM and PCR in the diagnosis of malaria in Cameroon was compared in order to illustrate the number of cases missed by traditional methods (clinical diagnosis, RDT and TFM) of diagnosis.

\section{Methods \\ Study area}

This study was conducted in three Regions of Cameroon, Far North, Centre and North West with varied climatic conditions and altitudes (Table 1). In the Far North Region with seasonal malaria transmission the study was conducted in Maroua $\left(10.5925^{\circ} \mathrm{N}, 14.3210^{\circ} \mathrm{E}\right)$ in October 2014. In the Central Region, which is holoendemic for malaria, the study was carried out in Nkolbisson (a neighbourhood in Yaoundé $\left(3.8480^{\circ} \mathrm{N}, 11.5021^{\circ} \mathrm{E}\right)$ from

Table 1 General characteristics of study population, study sites and environmental factors

\begin{tabular}{|c|c|c|c|c|}
\hline \multirow[t]{2}{*}{ Characteristics } & \multicolumn{3}{|l|}{ Study sites } & \multirow[t]{2}{*}{ Total, n (\% } \\
\hline & Maroua, n (\%) & Nkolbisson, n (\%) & Bamenda, n (\%) & \\
\hline \multicolumn{5}{|l|}{ Gender } \\
\hline Male & $64(52)$ & $168(53)$ & $33(29)$ & $265(48)$ \\
\hline Female & $60(48)$ & $147(47)$ & $79(71)$ & $286(52)$ \\
\hline Total & 124 & 315 & 112 & 551 \\
\hline \multicolumn{5}{|l|}{ Age group (years) } \\
\hline $0-5$ & $81(65)$ & $180(57)$ & $34(30)$ & $295(54)$ \\
\hline $6-10$ & $15(12)$ & $86(27)$ & $5(4)$ & $106(19)$ \\
\hline $11-16$ & $9(7)$ & $49(16)$ & $4(4)$ & $62(11)$ \\
\hline$\geq 17$ & $19(15)$ & $0(0)$ & $69(61)$ & $88(16)$ \\
\hline Environmental factors [10] & Maroua & Nkolbisson & Bamenda & \\
\hline Climate & Sahel & Tropical & Tropical & \\
\hline Average annual temperature $\left({ }^{\circ} \mathrm{C}\right)$ & 28.3 & 23.7 & 21.5 & \\
\hline Average annual rainfall (mm) & 794 & 1643 & 2145 & \\
\hline Elevation (m) & 384 & 750 & 1614 & \\
\hline $\begin{array}{l}\text { Weather condition at time of specimen } \\
\text { collection }\end{array}$ & End of rainy season & Rainy season & Dry season & \\
\hline
\end{tabular}


February 2014 to April 2014. In the North West Region, which is holoendemic for malaria, the study was conducted in Bamenda $\left(5.9631^{\circ} \mathrm{N}, 10.1591^{\circ} \mathrm{E}\right)$ in February 2015.

\section{Study design}

A cross-sectional study was conducted in selected health facilities in Maroua, Nkolbisson, and Bamenda. Inclusion criteria were age $>6$ months and axillary temperature $>37.5{ }^{\circ} \mathrm{C}$ at the time of recruitment or fever within $24 \mathrm{~h}$ preceding recruitment. A written informed consent was obtained from all study participants $\geq 18$ years of age. Parents or legal guardians of children $<18$ years gave a written informed consent on behalf of their children.

\section{Ethical considerations}

Ethical approvals were obtained from the Committee on Human Subjects of the University of Hawaii (protocol number CHS 21724) and the National Research Ethics Committee of the Ministry of Public Health, Cameroon (protocol number 2014/04/442/CE/CNERSH/SP). Administrative approvals were obtained from the Ministry of Public Health, Cameroon and the Directors of the participating facilities.

\section{Study procedures}

An easy-to-read questionnaire was used for the collection of demographic and clinical data. After obtaining informed consent, the research or clinic staff took axillary temperatures and recorded the reported signs and symptoms. Venous blood, $2-5 \mathrm{~mL}$, was collected from each participant, dispensed into ethylenediaminetetraacetic acid (EDTA) tubes and stored in cold boxes until transported to the research laboratory where they were stored at $4-8{ }^{\circ} \mathrm{C}$. TFM and RDT for malaria were conducted for all participants, and both results were presented to the consulting health care worker (HCW). At the end of the hospital visit, an exit survey was conducted to obtain information on (1) the clinical diagnosis made by the $\mathrm{HCW}$, and (2) determine if anti-malarial drugs had been prescribed. This information was used to determine how frequent anti-malarial drugs were used to treat malaria-negative patients. When exit survey information was not available, the hospital record was consulted for the information.

\section{Laboratory investigations Malaria TFM}

Thick blood films were prepared and stained using 10\% Giemsa for $15 \mathrm{~min}$. A slide was considered positive if at least one asexual blood-stage $P$. falciparum parasite was identified. Parasitaemia was determined by counting the number of parasites per 200 white blood cells and assuming that each subject had 8000 white blood cells $/ \mu \mathrm{L}$ of blood. Two readings were conducted for each slide and discrepancies greater than $10 \%$ were resolved by a third reading by an independent trained microscopist. The slides for this study were read by well-trained and experienced scientists who have worked in malaria-research for a number of years.

\section{Malaria RDT}

Approximately $5 \mu \mathrm{L}$ of blood was used to diagnose malaria using the Ag Pf/Pan malaria RDT kit (Standard Diagnostic Inc., South Korea), following the manufacturer's instructions. This RDT is a qualitative immunochromatographic test that detects P. falciparum HRP-II and Plasmodium lactate dehydrogenase, which is a glycolytic enzyme common to $P$. falciparum, Plasmodium ovale, Plasmodium vivax and Plasmodium malariae asexualstage parasites.

\section{Malaria PCR}

DNA was extracted from $200 \mu \mathrm{L}$ of whole blood using the mini-prep spin-column technique (MachereyNagel, Germany) following the manufacturer's instructions. Detection of malaria parasite DNA was based on nested PCR amplification of the $18 \mathrm{~s}$ rRNA gene in a reaction that used $2 \mu \mathrm{L}$ of the extracted DNA, $10 \mu \mathrm{L}$ of GoTaq polymerase and master mixes (Promega, USA), $0.25 \mu \mathrm{M}$ each of upstream and downstream primers, and $6 \mu \mathrm{L}$ of nuclease free water in a total reaction volume of $20 \mu \mathrm{L}$. The first PCR encompassed genus-specific primers and the second nested PCR run encompassed the species-specific primers for $P$. falciparum, as previously described [9]. The presence of a characterizing band of $\sim 205$-bp for $P$. falciparum visualized on a UV transilluminator after electrophoresis on a $2 \%$ agarose gel stained with ethidium bromide.

\section{Statistical analysis}

Data were entered into Microsoft Office Excel and analysed using StatPlus 5.9.80 (AnalystSoft Inc., Walnut, CA) and Prism 6.0 (Graphpad Software, San Diego, CA) for descriptive statistics. Diagnostic test performance for clinical diagnosis, TFM and RDT for the diagnosis of malaria was analysed using MedCalc 16.8 (Ostend, Belgium). Descriptive statistics are represented as frequencies and medians. Sensitivity, specificity, positive and negative predictive values, accuracy and percentage of agreement (kappa value) were calculated with confidence intervals by age groups. Multivariable logistic regression was conducted to identify correct diagnosis comparing other test methods (TFM and RDT) to PCR. $P<0.05$ was considered statistically significant. All the other analyses were conducted using SAS version 9.4 (SAS Institute 
Inc., Cary, NC). The figure was generated using $\mathrm{R}$ version 3.4.2.

\section{Results}

General characteristics of the study population and study site environmental factors

Of the 551 febrile patients recruited from the selected health care facilities in Cameroon, 57\% resided in Nkolbisson; 23\% in Maroua and, 20\% in Bamenda. The overall distribution of males and females was $48 \%$ and $52 \%$, respectively. The majority of the study participants were in the age-group of $0-5$ years, with $81(65 \%)$ in this age group in Maroua, and 180 (57\%) in Nkolbisson. In Bamenda however, 69 (61\%) of the study participants were over 17 years of age (Table 1 ).

\section{Proportion of diagnosed malaria cases by various diagnostic methods}

The overall proportion of participants diagnosed with malaria by TFM, RDT, and PCR in the cohort was $31 \%$, $45 \%$, and $54 \%$, respectively. However, $92 \%$ (507/551) of the participants were diagnosed with clinical malaria (temperature range 36.5 to $39.7{ }^{\circ} \mathrm{C}$ ), of which $41 \%$ $(209 / 551)$ were negative for malaria by PCR (Table 2).
All the participants (507) who were diagnosed with clinical malaria were prescribed anti-malarial drugs by the HCW. The prevalence of malaria by PCR in Maroua, Nkolbisson, and Bamenda was 57\%, 70\% and 5\%, respectively. Therefore, a proportion of malaria cases were missed by TFM and RDT. Unfortunately, $41 \%$ of patients were falsely diagnosed as being positive for malaria by $\mathrm{HCW}$.

\section{Sub-microscopic infections detected by RDT and PCR}

Sub-microscopic $P$. falciparum infections were defined as i) positive by PCR but negative by microscopy, ii) positive by PCR but negative by RDT, and iii) positive by RDT but negative by TFM. A total of 128 (23\%) febrile patients were positive for $P$. falciparum by PCR, but negative by TFM (Table 3). The lowest parasitaemia detected by TFM was 120 parasites/ $\mu \mathrm{L}$ of blood. Meanwhile, $65(12 \%)$ febrile patients were positive for P. falciparum by PCR and negative by RDT. However, 84 (15\%) patients were positive for malaria by RDT but negative by PCR. This shows that, $23 \%$ of malaria cases were missed by TFM while RDT detected $15 \%$ more malaria infections compared to TFM but failed to detect $12 \%$ detected by PCR.

Table 2 Malaria prevalence stratified by test method and study site

\begin{tabular}{lclll}
\hline Test method & \multicolumn{2}{l}{ Study sites } & & Total $(\mathbf{n = 5 5 1 )}$ \\
\cline { 2 - 5 } & Maroua $(\mathbf{n = 1 2 3 )}$ & Nkolbisson $(\mathbf{n = 3 1 5})$ & Bamenda $(\mathbf{n}=\mathbf{1 1 2})$ & $\mathbf{M P + ( \% )}$ \\
\hline MP+ (\%) & MP+ (\%) & MP+ (\%) & $172(31)$ \\
TFM & $32(26)$ & $135(43)$ & $5(4)$ & $249(45)$ \\
RDT & $67(54)$ & $175(55)$ & $7(6)$ & $298(54)$ \\
PCR & $71(57)$ & $221(70)$ & $6(5)$ & $507(92)$ \\
Clinical diagnosis & $120(97)$ & $301(96)$ & $86(77)$ & \\
\hline
\end{tabular}

Table 3 Prevalence of sub-microscopic $P$. falciparum infection by age-group

\begin{tabular}{|c|c|c|c|c|}
\hline \multirow[t]{2}{*}{ Age-group $^{a}(n)$} & \multirow{2}{*}{$\begin{array}{l}\text { Median parasites } / \mu \mathrm{L} \text { (range: } \\
\text { Q1-Q3) }\end{array}$} & \multicolumn{3}{|l|}{ Test characteristic } \\
\hline & & $\begin{array}{l}\text { PCR (+), TFM (-) } \\
\text { n (\%) }\end{array}$ & $\begin{array}{l}\text { PCR (+), RDT (-) } \\
\text { n (\%) }\end{array}$ & $\begin{array}{l}\text { RDT (+), TFM (-) } \\
\text { n (\%) }\end{array}$ \\
\hline $0-5(295)$ & $12,405(1500-300,000)$ & $72(28)$ & $45(17)$ & $37(14)$ \\
\hline $6-10(106)$ & $15,200(3200-300,000)$ & $34(32)$ & $12(11)$ & $27(25)$ \\
\hline $11-16(62)$ & $8640(3360-75,200)$ & $16(26)$ & $5(8)$ & $13(21)$ \\
\hline$\geq 17(88)$ & $721(461-852)$ & $6(7)$ & $3(3)$ & $7(8)$ \\
\hline Total $(n=551)$ & & $128(23)$ & $65(12)$ & $84(15)$ \\
\hline
\end{tabular}

PCR (+), TFM (-) $=$ PCR positive but TFM negative

$\mathrm{PCR}(+), \mathrm{RDT}(-)=\mathrm{PCR}$ positive but RDT negative

RDT $(+)$, TFM $(-)=$ RDT positive but TFM negative

Q1-Q3: 25\% to $75 \%$ interquartile range

TFM thick film microscopy, $R D T$ rapid diagnostic test, $P C R$ polymerase chain reaction

a In years 


\section{Proportion of diagnosed malaria cases by test method in different age group}

Across the different age-groups, RDT and PCR detected more malaria infections as compared to TFM (Fig. 1). Moreover, the age-specific detection of malaria by RDT was closer to that of PCR. However, the rate of clinical diagnosis of malaria was high irrespective of the agegroup. There was no significant difference in accurately diagnosing malaria by RDT (95\% CI $0.52-2.57 ; \mathrm{p}=0.23$ ) and TFM (95\% CI 0.77-2.96; $\mathrm{p}=0.73$ ) between those aged $\leq 5$ years and $>5$ years.

Also, across the different age-groups, the sensitivity and specificity of TFM and RDT were similar (Table 4). This means that across different age-groups, RDT was almost as good as PCR, but still missed $12 \%$ of malaria cases. Also, both RDT and TFM work well across different age-groups.

\section{Comparison of diagnostic accuracy of clinical diagnosis, TFM and RDT vs. PCR}

Using PCR as reference standard, 298 participants were positive for malaria while 253 participants were negative for malaria (Table 5). RDT correctly identified 234 (79\%) infections (true positive), microscopy correctly identified 170 (57\%) infections; whereas clinical diagnosis identified all 298 (100\%) infections. However, there were 209 (83\%) false positive clinical diagnosis, $15(6 \%)$ by RDT and $2(0.8 \%)$ by TFM. The sensitivity of TFM, RDT, and

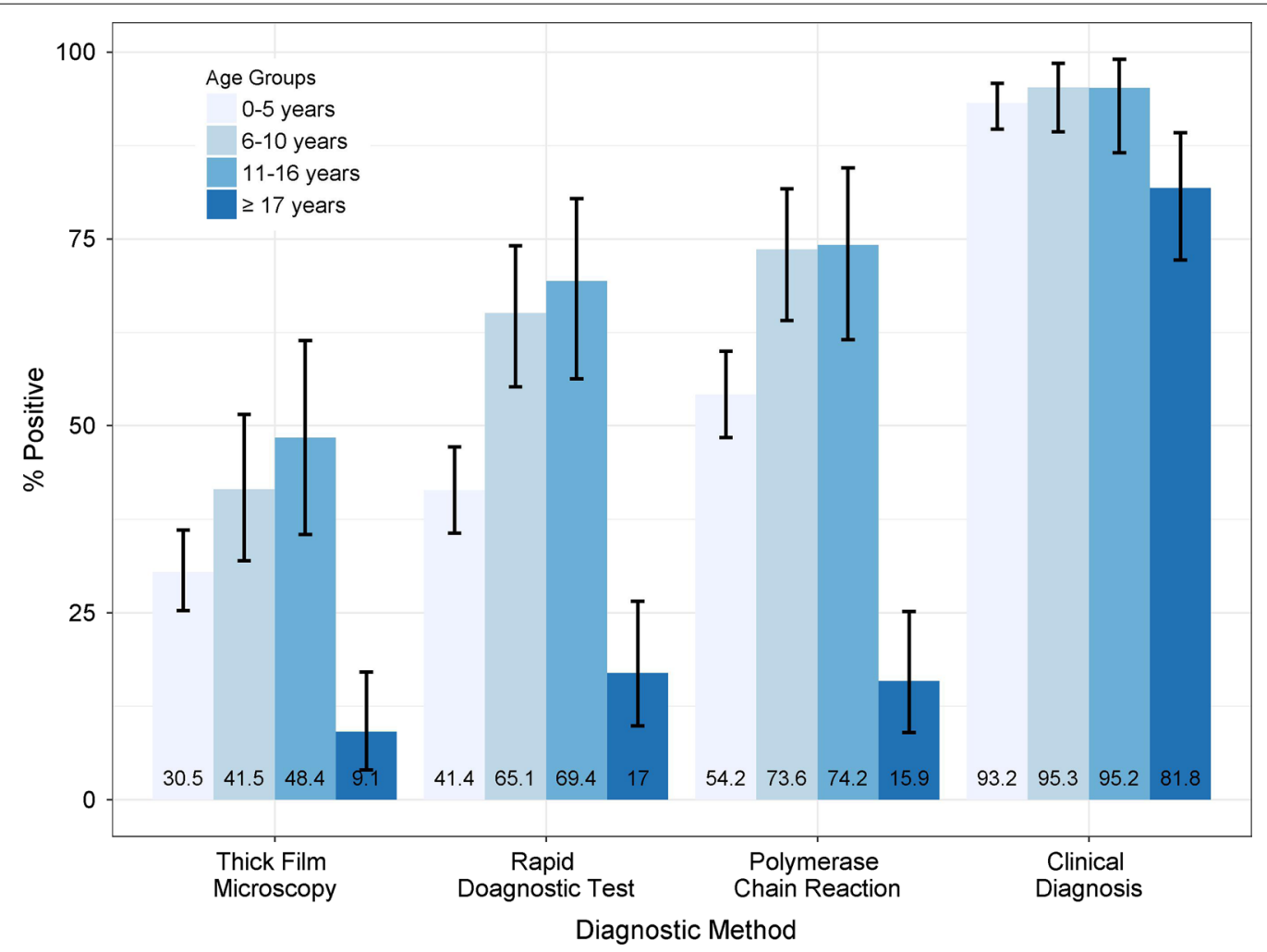

Fig. 1 Age-specific detection of malaria by diagnostic method

Table 4 Performance of TFM and RDT across age-groups with PCR as reference method

\begin{tabular}{lcccc}
\hline Age group (years) & \multicolumn{1}{c}{ Sensitivity TFM (95\% Cl) } & Specificity TFM (95\% Cl) & Sensitivity RDT (95\% Cl) & $\begin{array}{l}\text { Specificity } \\
\text { RDT (95\% Cl) }\end{array}$ \\
\hline $0-5$ & & $98 \%(95-99)$ & $72 \%(64-79)$ & $95 \%(90-98)$ \\
$6-10$ & $55 \%(45-63)$ & $100 \%(88-100)$ & $85 \%(75-92)$ & $89 \%(72-98)$ \\
$11-16$ & $56 \%(45-68)$ & $100 \%(79-100)$ & $89 \%(76-96)$ & $87 \%(62-98)$ \\
$\geq 17$ & $65 \%(50-79)$ & $100 \%(95-100)$ & $79 \%(49-95)$ & $95 \%(87-98)$ \\
\hline
\end{tabular}

TFM thick film microscopy, $R D T$ rapid diagnostic test, $P C R$ polymerase chain reaction, $C l$ confidence interval 
Table 5 Diagnostic test performance of clinical diagnosis, RDT, and TFM in the diagnosis of malaria with PCR as reference method

\begin{tabular}{llll}
\hline Test characteristic & Clinical diagnosis & RDT & TFM \\
\hline TP (PCR $=298)$ & 298 & 234 & 170 \\
FP (PCR negative) & 209 & 15 & 2 \\
TN (PCR $=253)$ & 44 & 237 & 251 \\
FN (PCR positive) & 0 & 65 & 128 \\
Sensitivity [95\% CI] & $100 \%[99-100]$ & $78 \%[73-82]$ & $57 \%[51-63]$ \\
Specificity [95\% CI] & $17 \%[13-23]$ & $94 \%[90-97]$ & $99 \%[97-99]$ \\
PPV [95\% CI] & $59 \%[54-63]$ & $94 \%[90-96]$ & $99 \%[96-99]$ \\
NPP [95\% Cl] & $100 \%[92-100]$ & $78 \%[73-83]$ & $66 \%[61-71]$ \\
Accuracy [95\% Cl] & $62 \%[58-66]$ & $85 \%[82-88]$ & $76 \%[73-80]$ \\
Kappa value $[95 \%$ Cl] & $0.18[0.14-0.24]$ & $0.71[0.65-0.77]$ & $0.54[0.48-0.60]$ \\
Misclassification rate $(\%)$ & 34 & 14 & 23 \\
\hline
\end{tabular}

$T P$ true positive, $F P$ false positive, $T N$ true negative, $F N$ false negative, $P P V$ positive predictive value, $N P V$ negative predictive value

clinical diagnosis was $57 \%, 78 \%$, and $100 \%$, respectively; the specificity was $99 \%, 94 \%$, and $17 \%$, respectively; the positive predictive value was $99 \%, 94 \%$, and $59 \%$, respectively, and negative predictive value was $66 \%, 78 \%$, and $100 \%$, respectively. The clinical diagnosis had a "poor" agreement (kappa 0.18), malaria RDT had a "good" agreement (kappa 0.71), and malaria microscopy had a "moderate" agreement (kappa 0.54) when compared to PCR. In general, the accuracy of clinical diagnosis was $62 \%$, RDT 85\%, and TFM 76\%. However, the error rate of clinical diagnosis was $34 \%$, RDT was $14 \%$, and TFM was $23 \%$ (Table 5).

\section{Discussion}

Accurate and prompt diagnosis of malaria is the only way to effectively treat, manage and eventually eliminate the disease. This study was conducted to determine the proportion of malaria cases missed by conventional malaria diagnostic methods, namely, TFM, RDT, clinical diagnosis, but detected by PCR.

In this study, TFM missed 23\% of PCR-positive malaria infections. In a previous meta-analysis based on data from 42 studies, microscopy missed about $50 \%$ of PCRpositive malaria infections [11]. Also, in a large epidemiological study in Cambodia a significant proportion of microscopy-negative samples were detected by PCR (289/7491; 3.85\%) [12]. False-negative microscopy results are known to increase as parasite density decreases [13]. Moreover, the detection threshold of Giemsa-stained TFM varies considerably between $50-500$ parasites/ $\mu$ l of blood. However, TFM predicted the presence of malaria parasite in $99 \%$ of the study participants making TFM a good "rule in" test for malaria. This means that a positive TFM result for malaria can be trusted; meanwhile, a negative result does not exclude the presence of malaria infection.

Malaria RDT missed $12 \%$ of PCR-positive malaria infections. However, the accuracy of malaria RDT was good as compared to PCR. The sensitivity and specificity of malaria RDT in this study was $78 \%$ and $94 \%$ respectively, which is consistent with a recent study in Kenya [14] that evaluated the same RDT. Several factors have been demonstrated to affect the sensitivity of RDTs based on detection of HRP-II, including an inherent limitation of the device, mutation or deletion of the gene encoding the HRP-II, and storage conditions $[13,15]$. Interestingly, RDT detected $15 \%$ of malaria cases that were missed by TFM. This is because RDT detects antigens, not parasites, which gives it an added advantage over microscopy in its ability to diagnose malaria in patients with low parasitaemia below the detection threshold of microscopy [7, 8]. However, there is the possibility of false positive RDT results when the malaria parasite is cleared, and antigens remain in circulation. In the present study, malaria RDT could predict the presence of malaria parasite in $94 \%$ of the study participants making it a good "rule in" test for malaria. Therefore, in the absence of PCR, malaria RDT can be used to improve the quality of care by ensuring appropriate treatment of confirmed malaria cases while avoiding indiscriminate administration of anti-malarial drugs for malaria-negative patients. However, because a substantial proportion of infections were missed by RDT, it is therefore not sufficiently sensitive for mass screening programmes as recommended by the WHO [16].

This study found high rates of clinical diagnosis and overtreatment of malaria, in all three Cameroonian study sites, which is consistent with previous studies [17-20]. Even though the sensitivity of clinical diagnosis of malaria was high, the overall accuracy of clinical 
diagnosis was poor with $41 \%$ of malaria-negative patients erroneously treated for malaria. Moreover, clinical diagnosis of malaria could predict the presence of malaria parasite in only $59 \%$ of study participants. Clearly, many patients that were treated for malaria had other causes of fever. Therefore, clinical diagnosis of malaria cannot be relied upon as a "rule in" test for malaria due to overlapping malaria symptoms with other tropical febrile illnesses.

Results of this study also provide information on the prevalence of malaria in three Regions of Cameroon. The prevalence of malaria was high in Nkolbisson and Maroua, but low in Bamenda. These three regions are characterized by different climatic variables (Table 1), which have been shown to affect the prevalence of malaria in a given region [21-25]. A previous study in Tanzania reported malaria prevalence proportions of $79-90 \%$, $27-46 \%$ and $8-16 \%$ in low, intermediate and high altitudes, respectively [26]. Results of this study thus provide information on the prevalence of malaria in three climatically different regions of Cameroon, which is important to guide malaria control interventions.

This study has a limitation in that quantitative PCR was not conducted in order to quantify parasitaemia. Thus, it was not possible to stratify the infections that were missed by TFM and RDT by level of parasite density in order to determine the variation in sensitivity across parasite density levels.

\section{Conclusions}

Results of this study suggest that the conventional diagnostic methods for malaria (TFM, RDT, ) are not adequate when accurate epidemiological data are needed to monitor malaria control and elimination interventions. PCR permitted the detection of $23 \%$ of malaria cases missed by TFM and RDT further, confirming it as a valuable tool for epidemiological surveys, mass screening, and the assessment of interventions for malaria elimination. Therefore, the development and standardization of a rapid and sensitive molecular-based test capable of detecting sub-microscopic malaria infection are warranted for the global elimination of malaria. Furthermore, continual training and proficiency testing should be instituted for laboratory technicians on malaria microscopy and post-market surveillance to assure the quality of malaria RDT since high-performance microscopy and quality assured RDTs will suffice for the clinical management of patients with suspected malaria.

\section{Abbreviations}

HRP-2: histidine-rich protein-II; nPCR: nested PCR; NLR: negative likelihood ratio; NPV: negative predictive value; PCR: polymerase chain reaction; PLR: positive likelihood ratio; PPV: positive predictive value; RDT: rapid diagnostic test; HCW: Health Care Workers; TFM: thick film microscopy.

\section{Authors' contributions}

$\mathrm{KOM}, \mathrm{VRN}, \mathrm{RGL}$ and OAA designed the study. KOM, OAA, MC, ONB, LFE COllected the samples, and conducted the laboratory studies. KG, KOM, VRN, DWT contributed to data analysis. KOM wrote the draft manuscript, DWT, VRN edited the manuscript, and all authors reviewed the final manuscript. RGL, DWT and VRN supervised the study. All authors read and approved the final manuscript.

\section{Author details}

1 Department of Tropical Medicine, Medical Microbiology and Pharmacology, John A. Burns School of Medicine, University of Hawaii at Manoa, Honolulu, HI, USA. ${ }^{2}$ Pacific Center for Emerging Infectious Diseases Research, John A. Burns School of Medicine, University of Hawaii at Manoa, Honolulu, HI, USA. ${ }^{3}$ Biotechnology Center, University of Yaoundé I, Yaoundé, Cameroon. ${ }^{4}$ National Medical Research Institute (IMPM), Yaoundé, Cameroon. ${ }^{5}$ Biostatistics Core Facility Department of Complementary \& Integrative Medicine, John A. Burns School of Medicine, University of Hawaii at Manoa, Honolulu, HI, USA.

\section{Acknowledgements \\ All study participants and Directors of the various healthcare facilities.}

\section{Competing interests}

The authors declare they have no competing interests.

\section{Availability of data and materials}

The dataset produced by the current study is available from the corresponding author upon request.

\section{Consent for publication}

Not applicable.

\section{Ethical approval and consent to participate}

Ethical approvals were obtained from the Committee on Human Subjects of the University of Hawaii (protocol number CHS 21724) and the National Research Ethics Committee of the Ministry of Public Health Cameroon (protocol number 2014/04/442/CE/CNERSH/SP). Administrative approvals were also obtained from the directors of the various health institutions and from the Ministry of Public Health, Cameroon.

\section{Funding}

KOM, LFE, VRN, DWT, RGL were supported by a Grant (5D43TW009074) from the Global Infectious Diseases Training Program, Fogarty International Center (FIC), National Institutes for Health (NIH) and the National Medical Research Institute (IMPM), Yaoundé, Cameroon. OAA and VRN were supported by $\mathrm{NIH}$ Research Training Grant (R25TW009345 and D43TW009345) awarded to the Northern Pacific Global Health Fellows Program by the FIC, NIH, USA. KG was partially supported by NIH Grant U54MD007584. Partial support was provided by a Grant (P30GM11473) from the Centers of Biomedical Research Excellence, National Institute of General Medical Sciences, NIH.

\section{Publisher's Note}

Springer Nature remains neutral with regard to jurisdictional claims in published maps and institutional affiliations.

Received: 23 November 2018 Accepted: 6 March 2019

Published online: 12 March 2019

\section{References}

1. WHO. World malaria report 2016. Geneva: World Health Organization; 2016.

2. WHO. Universal access to malaria diagnostic testing. An operational manual. Geneva: World Health Organization; 2013.

3. Perkins MD, Bell DR. Working without a blindfold: the critical role of diagnostics in malaria control. Malar J. 2008;7(Suppl 1):S5. 
4. Chandler Cl, Mangham L, Njei AN, Achonduh O, Mbacham WF, Wiseman $V$.'As a clinician, you are not managing lab results, you are managing the patient': how the enactment of malaria at health facilities in Cameroon compares with new WHO guidelines for the use of malaria tests. Soc Sci Med. 2012;74:1528-35.

5. Ye Y, Madise N, Ndugwa R, Ochola S, Snow RW. Fever treatment in the absence of malaria transmission in an urban informal settlement in Nairobi. Kenya. Malar J. 2009;8:160.

6. Mangham LJ, Cundill B, Achonduh OA, Ambebila JN, Lele AK, Metoh TN, et al. Malaria prevalence and treatment of febrile patients at health facilities and medicine retailers in Cameroon. Trop Med Int Health. 2012;17:330-42

7. Batwala V, Magnussen P, Nuwaha F. Are rapid diagnostic tests more accurate in diagnosis of plasmodium falciparum malaria compared to microscopy at rural health centres? Malar J. 2010;9:349.

8. Azikiwe CC, Ifezulike CC, Siminialayi IM, Amazu LU, Enye JC, Nwakwunite OE. A comparative laboratory diagnosis of malaria: microscopy versus rapid diagnostic test kits. Asian Pac J Trop Biomed. 2012;2:307-10.

9. Snounou G, Viriyakosol S, Zhu XP, Jarra W, Pinheiro L, do Rosario VE, et al. High sensitivity of detection of human malaria parasites by the use of nested polymerase chain reaction. Mol Biochem Parasitol. 1993:61:315-20.

10. Climate data for cities worldwide. https://en.climate-data.org. Accessed 20 March 2015.

11. Okell LC, Ghani AC, Lyons E, Drakeley CJ. Submicroscopic infection in Plasmodium falciparum-endemic populations: a systematic review and meta-analysis. J Infect Dis. 2009;200:1509-17.

12. Lek D, Popovici J, Ariey F, Vinjamuri SB, Meek S, Bruce J, et al. National malaria prevalence in Cambodia: microscopy versus polymerase chain reaction estimates. Am J Trop Med Hyg. 2016;95:588-94.

13. Wongsrichanalai C, Barcus MJ, Muth S, Sutamihardja A, Wernsdorfer WH. A review of malaria diagnostic tools: microscopy and rapid diagnostic test (RDT). Am J Trop Med Hyg. 2007;77:119-27.

14. Wanja EW, Kuya N, Moranga C, Hickman M, Johnson JD, Moseti C, et al. Field evaluation of diagnostic performance of malaria rapid diagnostic tests in western Kenya. Malar J. 2016;15:456.

15. Mouatcho JC, Goldring JPD. Malaria rapid diagnostic tests: challenges and prospects. J Med Microbiol. 2013:62:1491-505.
16. WHO. Policy brief on malaria diagnostics in low-transmission settings. Geneva: World Health Organization, WHO/HTM/GMP; 2014.

17. Chinkhumba J, Skarbinski J, Chilima B, Campbell C, Ewing V, San Joaquin $M$, et al. Comparative field performance and adherence to test results of four malaria rapid diagnostic tests among febrile patients more than five years of age in Blantyre. Malawi. Malar J. 2010;9:209.

18. Lubell $Y$, Reyburn $H$, Mbakilwa H, Mwangi $R$, Chonya S, Whitty CJ, et al. The impact of response to the results of diagnostic tests for malaria: costbenefit analysis. BMJ. 2008:336:202-5.

19. Yegorov S, Galiwango RM, Ssemaganda A, Muwanga M, Wesonga I, Miiro $\mathrm{G}$, et al. Low prevalence of laboratory-confirmed malaria in clinically diagnosed adult women from the Wakiso district of Uganda. Malar J. 2016:15:555.

20. Salomao CA, Sacarlal J, Chilundo B, Gudo ES. Prescription practices for malaria in Mozambique: poor adherence to the national protocols for malaria treatment in 22 public health facilities. Malar J. 2015;14:483.

21. Huang F, Zhou S, Zhang S, Wang H, Tang L. Temporal correlation analysis between malaria and meteorological factors in Motuo County. Tibet. Malar J. 2011;10:54.

22. Christiansen-Jucht CD, Parham PE, Saddler A, Koella JC, Basanez MG. Larval and adult environmental temperatures influence the adult reproductive traits of Anopheles gambiae s.s. Parasit Vectors. 2015:8:456.

23. Reiner RC Jr, Geary M, Atkinson PM, Smith DL, Gething PW. Seasonality of Plasmodium falciparum transmission: a systematic review. Malar J. 2015:14:343.

24. Sena L, Deressa W, Ali A. Correlation of climate variability and malaria: a retrospective comparative study. Southwest Ethiopia. Ethiop J Health Sci. 2015;25:129-38.

25. Tchuinkam T, Nyih-Kong B, Fopa F, Simard F, Antonio-Nkondjio C, AwonoAmbene HP, et al. Distribution of Plasmodium falciparum gametocytes and malaria-attributable fraction of fever episodes along an altitudinal transect in Western Cameroon. Malar J. 2015;14:96.

26. Bødker R, Akida J, Shayo D, Kisinza W, Msangeni HA, Pedersen EM, et al. Relationship between altitude and intensity of malaria transmission in the Usambara Mountains, Tanzania. J Med Entomol. 2003;40:706-17.

Ready to submit your research? Choose BMC and benefit from:

- fast, convenient online submission

- thorough peer review by experienced researchers in your field

- rapid publication on acceptance

- support for research data, including large and complex data types

- gold Open Access which fosters wider collaboration and increased citations

- maximum visibility for your research: over $100 \mathrm{M}$ website views per year

At BMC, research is always in progress.

Learn more biomedcentral.com/submissions 